\title{
Experiencia universitaria innovadora sobre didáctica de la motricidad infantil: De las aulas universitarias a las aulas de Educación Infantil
}

\author{
Innovative Experience Related to Didactics of Early Childhood Motricity: From University Classroom \\ to Early Childhood Education Classroom
}

\section{Experiência universitária inovadora no ensino das habilidades motoras infantis: das salas de aulas da universidade às salas de aula de educação infantil}

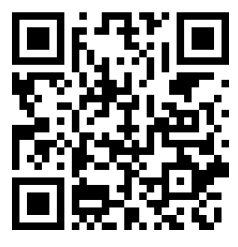

Vladimir Martínez-Bello

Universitat de València

Cuerpo, Movimiento, Música y Prácticas Curriculares

Valencia, España

vladimir.martinez@uv.es

http://orcid.org/0000-0002-3408-9678

María del Mar Bernabé-Villodre

Universitat de València

Cuerpo, Movimiento, Música y Prácticas Curriculares

Valencia, España

maria.mar.bernabe@uv.es

http://orcid.org/0000-0001-8983-6602

Recibido • Received • Recebido: 14 / 12 / 2017

Corregido • Revised • Revisado: 18 / 02 / 2019

Aceptado • Accepted • Aprovado: 20/ 05 / 2019

\begin{abstract}
Resumen: La relación entre universidad y aulas de Educación Infantil parece concentrarse durante el Prácticum, y queda en el olvido una posible interrelación del currículo universitario con la realidad educativa en otras oportunidades de la formación universitaria. El objetivo del estudio fue el análisis de la implementación de una experiencia innovadora en el grado de Maestra o Maestro en Educación Infantil, mediante la participación de docentes de Educación Infantil, estudiantes y profesorado universitario. El estudiantado universitario fue dotado de un corpus teórico relativo a las principales perspectivas curriculares sobre la motricidad infantil, en el marco de la asignatura Didáctica de la Educación Física de la Educación Infantil (Universitat de Valencia, 2017a), complementada con formación práctica en movimiento musical. Se concertaron visitas a centros escolares para observar y reflexionar en relación con las sesiones de psicomotricidad en pequeños grupos. Las estudiantes elaboraron un documento de observación y reflexión en el que relacionaron los contenidos de las aulas universitarias con la experiencia en las aulas de psicomotricidad. Se destaca que la forma en que la maestra facilita la interacción mediante el uso de materiales, la atención a los contenidos referentes a las perspectivas teóricas, y la relación de conocimientos teóricos sobre la estructura de la sesión fueron algunas de las conclusiones elaboradas por el estudiantado. Se demuestra la necesidad de continuar innovando para acercar las aulas universitarias a los contextos educativos reales de las aulas de educación infantil. Finalmente, las visitas a los centros escolares para la observación y reflexión de sesiones de psicomotricidad infantil pueden favorecer la formación del profesorado en Educación Infantil.
\end{abstract}


doi: http://dx.doi.org/10.15359/ree.23-3.15

URL: http://www.una.ac.cr/educare

CORREO: educare@una.cr

Palabras claves: Educación infantil; enseñanza; psicomotricidad; universidad.

\begin{abstract}
The relationship between the university and early childhood classrooms seems to be the main focus during the practicum, leaving behind a possible interrelation between the university curriculum and the reality of education. The aim of the study was the analysis, evaluation, creation, and implementation of an experience focused on the didactics of child motor skills, through the participation of early childhood education teachers, university students, and university teachers. First, the students received a theoretical corpus on the main curricular perspectives of didactics of movement, from an innovative experience that unites psychomotor classes at Valencian schools with the subject Teaching of Physical Education of Early Childhood Education (Universitat de Valencia, 2017a), complemented with practical training in musical movement. After, the university students elaborated a report on their reflection and observations. In this document, they related the theoretical and practical knowledge from the university classroom with the psychomotricity experience in classrooms. One of the conclusions drawn was about how the teacher facilitates the interaction through the use of materials, the attention of the contents of theoretical perspectives, and the relation to the theoretical knowledge of the structure of the session; this conclusion was drawn from attending to the categories contextualized. We demonstrated the importance of innovating continuously; on the one hand, innovation is important to bring the university classrooms closer to the real education contexts of the early childhood education classrooms, and, on the other, to promote the teaching education process of early childhood education teachers.
\end{abstract}

Keywords: Preschool education; psychomotricity; teaching; university.

Resumo: A relação entre as salas de aula da universidade e as da educação infantil parece estar concentrada durante o Practicum, esquecendo-se da possivel inter-relação do currículo universitário com a realidade educacional como outras oportunidades da formação universitária. O objetivo dessa pesquisa foi analisar a implementação de uma experiência inovadora no curso de pedagogia para Educação Infantil, através da participação de professores de educação infantil, estudantes e docentes universitários. O grupo de e estudantes universitários foi dotado de um corpo teórico sobre as principais perspectivas curriculares relacionadas com habilidades motoras infantis, no âmbito da pedagogia para a Educação Física e a Educação Infantil (Universitat de Valencia, 2017a), complementado pela formação prática em movimento musical. Foram feitas visitas às escolas para observar e refletir sobre as sessões de psicomotricidade em pequenos grupos. Os alunos elaboraram um documento de observação e reflexão que relacionavam os conteúdos das salas de aula universitárias com a experiência nas salas de aula de psicomotricidade. A forma como a pessoa docente facilita a interação por meio do uso de materiais, a atenção aos conteúdos referentes às perspectivas teóricas e a relação dos conhecimentos teóricos sobre a estrutura da sessão foram algumas das conclusões elaboradas pelo grupo de estudantes. Isso demonstra a necessidade de continuar inovando para aproximar as salas de aula das universidades dos contextos educacionais reais de educação infantil. Finalmente, as visitas às escolas para observação e reflexão das sessões de psicomotricidade infantil podem favorecer a formação de professores para educação infantil.

Palavras-chave: Educação infantil; ensino; habilidades psicomotoras; universidade. 


\section{Introducción}

El documento de Propuestas para la renovación de las metodologías educativas en la universidad elaborado por el Ministerio de Educación y Ciencia (MEC, 2006) señala la necesidad de la renovación de las metodologías educativas en la universidad para atender a los nuevos desafíos de la universidad actual. En esta línea, el documento guía del proyecto Tuning (González y Wagenaar, 2003) estableció una serie de competencias básicas que debían ser alcanzadas en los procesos de enseñanza-aprendizaje de la educación superior. Para Ibarra y Rodríguez (2011), se concibe la competencia como un atributo latente, actitud, destreza, habilidad y facultad para el desarrollo de una actuación académica, ejecutándola adecuada y correctamente la actividad exigida. Como señaló Zabalza (2008), el enfoque por competencias indica un modelo de enseñanza en la universidad dirigida a fomentar la capacidad de actuación del estudiantado.

En este marco, se resaltan dos competencias generales del título de maestra o maestro en Educación Infantil en la Universidad de Valencia, que ayudarán a comprender el propósito de la divulgación de esta experiencia:"a) Promover y facilitar los aprendizajes en la primera infancia, desde una perspectiva globalizadora e integradora de las diferentes dimensiones cognitiva, emocional, psicomotora y volitiva" (Universitat de València, 2017b, p. 108); y“b) Comprender que la observación sistemática es un instrumento básico para poder reflexionar sobre la práctica y la realidad, así como contribuir a la innovación y a la mejora en educación" (Universitat de València, 2017b, p. 108). En este marco, la implantación de los títulos de grado en el Espacio Europeo de Educación Superior (EEES) debería promover el desarrollo y evaluación de competencias en la enseñanza universitaria más acorde con la realidad social que permea los procesos de enseñanza-aprendizaje, sobre todo, por ejemplo, con la implicación de un contacto más real con las aulas de los centros escolares en el currículo de formación del profesorado.

Por otra parte, la atención al desarrollo psicomotriz de las niñas y los niños tiene un espacio curricular importante en la formación del profesorado de Educación Infantil debido a que la educación motriz en el currículo de Educación Infantil juega un papel fundamental en el desarrollo y bienestar de la niñez (Bueno-Moral, Manchón-Ruíz y Moral-García, 1990; Conselleria de Educación, 2008; Howie et al., 2016; Silva-Moreira, Neves de Almeida y Moreira-Marinho, 2016; Teixeira-Costa, Abelairas-Gomez, Arufe-Giráldez, Pazos-Couto y Barcala-Furelos, 2015). No obstante, la formación de maestras y maestros competentes necesita que su formación en educación motriz se fundamente en el desarrollo de una actitud crítica sobre el papel que juega la educación motriz en las aulas escolares (Contell-Lahuerta, Molina-García y MartínezBello, Bernabé, Molina y Estevan, 2017; MEC, 2006). Sin embargo, como señala Rosales (2013), en la formación inicial del profesorado se hace necesaria una vivencia de las competencias a lo largo de todas las materias del grado, para evitar reproducir en el mundo laboral únicamente modelos trabajados en la formación inicial. Tal como señalan Álvarez y Hevia (2013), la educación superior en el nuevo modelo de educación superior en Europa propone una serie de desafíos 
doi: http://dx.doi.org/10.15359/ree.23-3.15

URL: http://www.una.ac.cr/educare

CORREO: educare@una.cr

para potenciar la relación teoría-práctica en la formación inicial del profesorado: plantean la búsqueda de vías diversas para conocer el sistema escolar y así poder acercarlo al alumnado en las aulas universitarias.

Por esa razón, esta innovación en el aula universitaria pretendió acercar la experiencia de maestras que trabajaban en el ámbito de la psicomotricidad infantil con el estudiantado que debía desarrollar competencias en el diseño y construcción de propuestas integradoras en el ámbito motriz, cognitivo y socioafectivo (Universitat de València, 2017a). Como previamente se señaló, tanto la competencia del desarrollo de la actitud crítica hacia el papel de la educación motriz en el currículo de la Educación Infantil como el de realizar propuestas integradoras en los ámbitos motriz, cognitivo y socioafectivo, son el sustento de este proyecto (UV-SFPIE_RMD16418238) aprobado por la Universidad de Valencia (Martínez-Bello et al., 2017).

La Universidad de Valencia forma parte de la red de universidades públicas del Estado español, aunque la comunidad autónoma donde se localiza (Comunidad Valenciana) otorga un especial carácter respecto a otras de distintas comunidades: el grado de competencia autonómica en materia educativa de esta comunidad es mayor que el de otras, lo que le otorga la posibilidad de un manejo y una disposición del currículo particulares. Esto mismo también puede observarse en los propios centros de Educación Infantil de esta misma comunidad autonómica.

En este marco, el presente estudio tiene la intención de presentar a la comunidad universitaria una experiencia educativa que describa cómo fue el proceso de implementación, evaluación y reflexión de un proyecto de innovación en una asignatura de un plan de estudios en una facultad de formación de profesorado de una universidad española. A continuación, se presentan los antecedentes teóricos y prácticas de experiencias innovadoras en el ámbito de la motricidad infantil para la formación del profesorado, y posteriormente se presenta el objetivo general y los objetivos específicos que impulsaron esta experiencia educativa.

\section{Experiencias de innovación realizadas en facultades de formación de profesorado}

Antes de realizar un recorrido sobre experiencias previas a la presente, debe mencionarse brevemente qué se entiende por innovación y por innovación en el aula universitaria, atendiendo a sus distintos enfoques en diferentes publicaciones en materia educativa, por supuesto. Pérez (2011) definió la innovación educativa como un proceso que debía acompañarse de las siguientes medidas: voluntad de la comunidad educativa, infraestructuras adecuadas, programas informáticos y formación de profesorado, principalmente. Aunque, sobre esa relación que suele establecerse entre innovación y necesaria presencia de tecnologías resultan interesantes las reflexiones de Vázquez (2016) quien nos invita a reconsiderar esto y a replantearnos si innovar educativamente es innovar tecnológicamente. En esta dirección, la presente experiencia innovadora daría fe de cómo la innovación educativa en la formación del profesorado no pasa necesariamente por la inclusión de tecnologías. 
Mérida, González y Olivares (2011) divulgaron los resultados de un proyecto de innovación en el cual se creó una red de colaboración entre tres instituciones: la universidad, las escuelas infantiles y el Centro de Formación Permanente del Profesorado. En el marco del proyecto, el estudiantado universitario visitó, en pequeños grupos, aulas de Educación Infantil de centros infantiles con el objetivo de mejorar el conocimiento de la metodología de investigación en las aulas de Educación Infantil conocida como los proyectos de trabajo. Las autoras encontraron que las estudiantes del grado de Magisterio en Educación Infantil desarrollaron competencias una vez terminada la experiencia innovadora, percibieron que las competencias más vinculadas fueron las destrezas relacionales respecto a la interacción con los niños y niñas, la realización de aportaciones personales y la colaboración con pares del pequeño grupo. De esta forma, confirmaron el papel que tiene este tipo de iniciativas de vincular la acción universitaria con la acción escolar, en favorecer la capacidad de interacción y comunicación entre los distintos actores.

Desde un artículo publicado hace 20 años por Vaca-Escribano y Medina-Vaquero (1997), no se tiene evidencia en el ámbito iberoamericano del impacto de la vivencia de estudiantado universitario respecto a su formación sobre la estructura de funcionamiento de sesiones vinculadas con el ámbito corporal y el movimiento en Educación Infantil. El objetivo de este estudio consistió en analizar una sesión práctica de educación corporal en aulas de educación infantil, realizada en colaboración conjunta entre estudiantado del segundo ciclo de Educación Infantil y profesorado universitario: realizaron un relato de la sesión con el objetivo de "construir el significado educativo de una práctica desde la interpretación y explicación compartida de lo ocurrido en ella" (Vaca-Escribano y Medina-Vaquero, 1997, p. 2). Del análisis de los relatos de los sujetos participantes, resaltaron: la estructura de funcionamiento, el desarrollo profesional, la identificación de nombre propios y la complejidad del ámbito corporal. Esto se constituyó en los diferentes informes que realizaron los sujetos participantes como producto del proceso de observación y reflexión de sesiones de educación corporal.

Más adelante, Vaca-Escribano (2000) compartió una experiencia innovadora realizada en el marco de un proyecto de colaboración conjunta entre profesorado de las escuelas y el profesorado universitario. Dicha experiencia tuvo como objetivo reflexionar sobre la forma en que se desarrollan las clases de motricidad, así como comprender las oportunidades de enseñanza y aprendizaje que la estructura de funcionamiento de la sesión posibilita. En dicha experiencia, participó profesorado universitario, profesorado de las escuelas que observaron y analizaron las prácticas curriculares en las sesiones de motricidad y que cada miembro, desde sus propios intereses y responsabilidades, compartió su visión sobre lo observado en esas sesiones.

En años posteriores, otras investigaciones se han centrado en conocer la percepción de distintos colectivos que integran el proceso educativo en relación con el papel de la motricidad en la Educación Infantil (Gil-Madrona, 2003). No obstante, para nuestro conocimiento, no existen experiencias recientes en el ámbito iberoamericano en las que se divulgue, por una parte, una 
doi: http://dx.doi.org/10.15359/ree.23-3.15

URL: http://www.una.ac.cr/educare

CORREO: educare@una.cr

experiencia educativa donde el estudiantado de una facultad de formación de profesorado y, en particular, del grado de Maestra o Maestro en Educación Infantil, visite y realice un proceso de interacción con el profesorado de aulas de educación infantil; $y$, a su vez, realice un proceso de observación para conocer cómo se llevan a cabo sesiones de motricidad infantil. Y, por otra parte, tampoco se cuenta con la realización de un proceso de reflexión sobre esas prácticas curriculares en función de los contenidos vividos en la asignatura de Didáctica de la Educación Física de la Educación Infantil. A continuación, se presentan los objetivos de la experiencia universitaria.

\section{Objetivos general y específicos}

El principal objetivo consistió en analizar y evaluar la puesta en práctica de una experiencia centrada en la didáctica de la motricidad infantil, mediante la participación de un grupo de maestras en ejercicio, de estudiantes y de profesorado universitario dentro de la asignatura de "Didáctica de la Educación Física de la Educación Infantil" del plan de estudios del grado de Maestra-o en Educación Infantil.Y, a nivel específico, se pretendieron los siguientes objetivos:

- Describir la forma en que el profesorado en ejercicio desarrollaba unidades didácticas de psicomotricidad infantil.

- Favorecer la interacción entre estudiantado de la asignatura "Didáctica de la Educación Física de la Educación Infantil" con profesorado que impartía las sesiones de psicomotricidad en aulas de Educación Infantil.

- Conocer la percepción de estudiantes de magisterio en Educación Infantil sobre la creación, implementación y evaluación de las sesiones de psicomotricidad infantil.

\section{Metodología}

\section{Generalidades}

La duración del proyecto fue de cuatro meses, durante el segundo cuatrimestre del curso académico 2016-2017. El proyecto recibió el visto bueno de la Comisión Académica de Título y fue aprobado por el Vicerrectorado de Políticas de Formación y Calidad Educativa de la Universidad de Valencia (proyecto: UV-SFPIE_RMD16-418238) (Martínez-Bello et al., 2017). Se recibió el visto bueno de la dirección de los centros escolares, así como del profesorado participante.

La experiencia que aquí se presenta tuvo sentido dentro del marco general de las competencias del Grado de Maestro en Educación Infantil de la Universidad de Valencia (Universitat de València, 2017a, 2017b), que fueron las siguientes: 
1. Comprender el concepto de didáctica de la educación física y su valor dentro el currículo en la etapa de Educación Infantil.

2. Desarrollar una actitud crítica hacia el papel de la educación motriz en el currículo de la Educación Infantil.

En particular, las competencias a desarrollar con este proyecto de innovación fueron:

1. Diseñar y utilizar espacios y entornos que permitan un desarrollo global de la motricidad del niño y la niña.

2. Realizar propuestas integradoras de los ámbitos motriz, cognitivo y socioafectivo, respetando las características de la etapa infantil.

A su vez, el proyecto de innovación se englobó, principalmente, con el desarrollo de los siguientes bloques de contenido de la guía docente de la asignatura "Didáctica de la Educación Física de la Educación Infantil". Los contenidos que se trabajaron especialmente fueron:

1. La educación motriz en el currículo de Educación Infantil: Análisis crítico y reflexión.

2. Didáctica de la motricidad en la Educación Infantil.

En este último bloque de contenidos, a su vez, aparecieron unidades importantes que debían considerarse:

1. Materiales y recursos didácticos.

2. Prácticas didácticas.

3. Las clases de educación física en el ámbito de la Educación Infantil: Organización, estructuración, planificación, metodología, estilos de enseñanza.

El estudiantado, al terminar el curso académico, debía haber desarrollado las siguientes competencias, claramente relacionadas con los objetivos establecidos para el proyecto y con su metodología de trabajo:

1. Diseñar y utilizar espacios y entornos que permitan un desarrollo global de la motricidad del niño o la niña.

2. Realizar propuestas integradoras de los ámbitos motriz, cognitivo y socioafectivo, respetando las características de la etapa infantil. 
doi: http://dx.doi.org/10.15359/ree.23-3.15

URL: http://www.una.ac.cr/educare

CORREO: educare@una.cr

Atendiendotodoesto,laexperienciapartiódeunaconcepcióninterpretativaconstructivista, en la que desde esa observación y recogida de información de profesorado activo y alumnado en formación en las propias aulas, se pudiese interpretar y comprender el fenómeno educativo y, no tanto, la explicación de sus causas. Ello permitió sustentar y construir la experiencia innovadora aquí presentada. Esta perspectiva se vio aumentada con los planteamientos de construcción de experiencias motrices en el aula universitaria, comprendiéndolas como paso previo para observar no participando la realidad del aula, y desarrollar, así, una perspectiva crítica ante el propio acto educativo de la motricidad en infantil y desde la formación universitaria recibida.

\section{Participantes}

Los sujetos participantes en el desarrollo de este proyecto fueron un grupo de estudiantes (16 chicos y chicas) de la Universidad de Valencia, muy implicados con los objetivos de este mismo y que cursaban las citadas asignaturas, y docentes (2 maestras) de diversos centros escolares públicos de la provincia de Valencia perteneciente a la Comunidad Valenciana (España). El alumnado universitario actuó como observador no participante, se limitó a la recogida de información, en atención a unos criterios que se comentarán en epígrafes siguientes. En cuanto al profesorado del aula de educación infantil, colaboraron prestando sus aulas para la observación de sus sesiones, explicaron previamente al alumnado universitario la estructura de sus sesiones $y$, finalmente, respondieron a una entrevista semi-estructurada. $Y$, respecto al personal formador universitario, prepara al alumnado en el aula a nivel práctico y teórico sobre lo que sucederá en el centro educativo y plantea el modo de trabajo para esta experiencia que se seguirá en el centro educativo.

La edad media de las participantes fue 25 años (DT = 4.20). En su gran mayoría fueron mujeres (98\%) mientras que hombres correspondió al 2\%. El 92\% de los sujetos participantes se dedicaba únicamente a estudiar en la universidad, el 6\% trabajaba como media 15 horas o más y el $2 \%$ trabajaba menos de 15 horas a la semana. En las visitas a los centros escolares, participaron en total seis grupos de 3-4 estudiantes. Asimismo, se contó con profesorado universitario (dos docentes más) implicado en las dos asignaturas mencionadas con anterioridad y que formaban parte del grupo del Proyecto referenciado (UV-SFPIE_RMD16-418238) (Martínez-Bello et al., 2017), constituido por un total de cuatro miembros. Por parte de los centros escolares, se contó con la participación de dos maestras en Educación Infantil pertenecientes a dos centros públicos, que estuvieron interesadas en colaborar en esta experiencia y cuya práctica psicomotora se ajustaba a los parámetros trabajados en el aula universitaria.

\section{Asignaturas implicadas}

Esta experiencia iniciada en las aulas universitarias, desde las asignaturas de "Didáctica de la Educación Física de la Educación Infantil" y "Procesos musicales en Educación Infantil", 
se extendió a las aulas de diversos colegios públicos de la provincia de Valencia (Comunidad Valenciana, España). Ambas asignaturas se constituyeron como bases de experiencia teórico-práctica para tomar consciencia de los elementos constituyentes de una sesión de psicomotricidad (algunas con elemento musical) y de las facilidades-dificultades de movimiento del niño ante determinadas exigencias motoras. En este último sentido, desde la asignatura de Música, se pretendió garantizar la consciencia corporal del propio estudiantado, como futuro personal docente de psicomotricidad, siguiendo la metodología de Jacques Dalcroze, que capacitaba al estudiantado para comprender su propio cuerpo y sus propias posibilidades/ limitaciones corporales ante el estímulo auditivo.

\section{Actividades previas realizadas en las aulas universitarias}

La asignatura de Educación Física tiene un bloque de contenidos titulado "Didáctica de la Motricidad en la Educación Infantil". En el marco de estos contenidos, el estudiantado habitualmente construye y evalúa sesiones de psicomotricidad que son puestas en práctica en el aula universitaria. Las sesiones de psicomotricidad se realizan en pequeños grupos (4 o 5 estudiantes) siguiendo lecturas sobre métodos y modelos de enseñanza que se trabajaban en educación infantil. Es habitual que, desde el inicio del cuatrimestre, cada grupo construyese una sesión (o sesiones) de psicomotricidad. Desde la asignatura de "Didáctica de la Educación Física de la Educación Infantil", el alumnado participó de diferentes momentos. Se explicaron los objetivos del proyecto, y se concretaron visitas a centros educativos públicos de la provincia de Valencia durante todo el segundo cuatrimestre. En estos, se trataba de conocer cómo se desarrollaban las sesiones de psicomotricidad de infantil, dentro del denominado segundo ciclo de Educación Infantil, único ciclo obligatorio en el Estado español.

El profesorado responsable de esta asignatura se reunió con los pequeños grupos (4 o 5 estudiantes) para explicar los objetivos del proyecto y para poder conocer su interés de participación en el mismo. Una vez constituidos los grupos, se procedió a determinar la primera reunión con el profesorado en ejercicio, en las aulas de educación infantil de los distintos centros públicos que se interesaron en participar de esta experiencia (concretamente, dos centros). La participación por parte del estudiantado fue voluntaria.

\section{Actividades realizadas en las aulas de psicomotricidad de educación infantil}

Con cada pequeño grupo se concertó una visita al centro educativo para conocer la forma en que se desarrollaban las sesiones de psicomotricidad en un aula de segundo ciclo de Educación Infantil. Para ello, se contó con la participación de algunas maestras en ejercicio (dos maestras), muy implicadas con el establecimiento de puntos de intercambio para facilitar la transición del aula universitaria al centro educativo. Posteriormente, se realizó una reunión entre 
doi: http://dx.doi.org/10.15359/ree.23-3.15

URL: http://www.una.ac.cr/educare

CORREO: educare@una.cr

los grupos de estudiantes, profesorado universitario y profesorado en ejercicio para proceder a construir conjuntamente una sesión de psicomotricidad, acorde con la realidad y las exigencias educativas (motoras, cognitivas, etc.) del alumnado de Educación Infantil.

Para la evaluación de las visitas a los centros escolares con el estudiantado, destinadas a observar y compartir una sesión de motricidad, se utilizaron dos momentos. Uno previo al inicio de la sesión, en donde se explicaron los lineamientos para realizar la observación y el posterior documento de evaluación. Estaba destinado a la evaluación de la experiencia educativa, de modo que el alumnado participante concentrase su atención en una persona estudiante al azar, para poder recoger las impresiones de su desarrollo motor a lo largo de cada sesión.

Y, en segundo lugar, la metodología de análisis de los documentos sobre la reflexión de la observación y su vinculación con las temáticas vistas en la asignatura de "Didáctica de la Educación Física de la Educación Infantil". En este marco, las preguntas fueron ideadas y construidas con base en la revisión de las principales fuentes bibliográficas de acuerdo con cinco características:

- La primera: se tomaron como referencia los principales bloques de contenidos de la Guía docente de la asignatura "Didáctica de la Educación Física de la Educación Infantil".

- La segunda: cada estudiante describió los patrones de juego y de actividad física del sujeto escogido al azar.

- La tercera: debían explicar, con base en la fase de descripción de los patrones de juego y de actividad física, la relación de la observación con los bloques de contenidos impartidos hasta ese momento durante la asignatura.

En definitiva, las directrices fueron expuestas a los sujetos participantes antes de comenzar la sesión de observación. Además, debían establecer relaciones con los contenidos teóricos de la asignatura; de forma que pudiesen distinguir cada momento de las diferentes sesiones de psicomotricidad presenciadas, que respondían a las principales perspectivas curriculares trabajadas en el aula universitaria.

\section{Actividad de cierre de la experiencia innovadora}

Una vez finalizada la experiencia educativa, se realizó una jornada de divulgación titulada "Jornada experiencia innovadora sobre psicomotricidad vivencial y control y conciencia corporal en educación infantil", que tuvo como objetivo socializar resultados provisionales del proyecto, y se contó con la participación de las maestras participantes de los centros escolares, el estudiantado participante y el profesorado universitario. La jornada fue abierta al público en general, y asistió profesorado escolar que manifestó su intención de vincularse para ampliar la 
red de participación en iniciativas de este tipo. La jornada contó con el apoyo del Servicio de Formación Permanente de la Universitat de València.

\section{Metodología de análisis de los documentos de reflexión del estudiantado}

Una vez finalizados los documentos de reflexión de las sesiones por parte del estudiantado participante en esta experiencia innovadora, se procedió a realizar un análisis cualitativo de los datos (Taylor y Bogdan, 1996). Para obtener una medida de la fiabilidad de la codificación, dos personas analizaron los textos de manera independiente (dos de los cuatro sujetos docentes implicados en el citado proyecto de innovación). Las categorías fueron construidas a posteriori con base en la detección de elementos en común en cada uno de las entrevistas. Una vez incorporados los documentos al programa QSR NVIVO 10 (QSR International Pty Ltd.), se elaboró una precategorización de la información. A través de este proceso, se detectaron los temas más significativos. De ese listado de temas, se comprobó la relación con las categorías teóricas que ayudaron a construir las preguntas de la entrevista realizada para finalizar el proceso. Una vez terminada la precategorización a través de una lectura independiente por parte de dos personas observadoras (nuevamente, dos de los cuatro sujetos docentes implicados en el proyecto, los mismos que la vez anterior), se procedió a la categorización final. Todo este proceso ha sido el que ha permitido afirmar que los datos obtenidos son válidos y fiables de cara a una mayor profundización en este enfoque, lo que quedaría como prospectiva.

Posteriormente, se reagrupó la información en cinco categorías, a saber:

- Respecto a la forma en que la maestra facilita la interacción a través del uso de materiales.

- Respecto a la relación de contenidos vistos en la universidad con la práctica real del aula.

- Respecto a la niña o niño que ha sido sujeto de la observación y la interacción de la estudiante con la maestra.

- Respecto a la detección de limitaciones de la sesión y posibles explicaciones.

- Respecto al agrado y utilidad de haber participado en la sesión de observación y reflexión.

Posteriormente, utilizando esas cinco categorías, se profundizó en el análisis de las categorías con mayor presencia de alusiones a los bloques de contenidos de la asignatura, vinculadas con la creación, ejecución y evaluación de las sesiones de psicomotricidad, por una parte; y, por otra, respecto a la detección de fortalezas y debilidades que, fruto de la reflexión, pueden ser mejoradas o reforzadas. 
doi: http://dx.doi.org/10.15359/ree.23-3.15

URL: http://www.una.ac.cr/educare

CORREO: educare@una.cr

En particular, respecto a la primera se extrajeron los siguientes resultados: la forma en que la maestra facilita la interacción a través del uso de materiales; la atención a los contenidos sobre las perspectivas teóricas, y la relación de conocimientos teóricos sobre la estructura de la sesión. Respecto a la segunda: limitaciones detectadas y posibles explicaciones respecto a la estructura y desarrollo de la sesión y reflexiones en torno a otros bloques de contenido de la asignatura "Didáctica de la Educación Física de la Educación Infantil".

\section{La percepción del profesorado involucrado}

Una vez terminada la visita a los centros escolares, las dos maestras de los centros escolares implicadas en el proyecto participaron en una entrevista semi-estructurada que fue grabada $y$, posteriormente, se procedió a su transcripción para analizar los resultados obtenidos.

\section{Resultados y discusión}

A continuación, se presentan las principales categorías extraídas del análisis del documento de evaluación de la visita a los centros escolares para la realización de la observación de las sesiones de psicomotricidad, que habían sido realizados por el estudiantado universitario participante de esta experiencia innovadora. Álvarez y Hevia (2013) señalaron que el nuevo sistema universitario en el marco del EEES ofrece una oportunidad de conocer ambos espacios y de trabajar conjuntamente, en mayor medida que con anteriores planes educativos. De esto se deduce que universidad y escuela deberían aprender a trabajar más conjuntamente como forma de evitar el divorcio entre espacios educativos que, anteriormente, ya habían comentado autoras como Blanco, Molina y López (2015), con una experiencia similar en Educación Primaria que trataba de conectar la realidad educativa con la formación inicial del personal docente; o la experiencia desde el prácticum de Mauri, Onrubia, Colomina y Clará (2016), centrada en promover la reflexión conjunta de maestras-futuras maestras-profesorado universitario. Así pues, esta experiencia innovadora se movió en ese mismo terreno en aras de conseguir una formación más completa, crítica y consecuente con la realidad actual.

En este marco, del análisis de los relatos del alumnado observador no participante en esas aulas visitadas pueden extraerse los siguientes resultados. A manera de ejemplo, se ha optado por, además de la categorización, exponer algunos ejemplos de las estudiantes que actuaron como observadoras no participantes.

\section{Respecto a la forma en que la maestra facilita la interacción a través del uso de materiales}

Las estudiantes son competentes para detectar que el papel de la maestra durante la sesión de psicomotricidad, a pesar de su carácter principalmente de observadora, esta facilita el desarrollo de la sesión, proponiendo nuevos retos y generando alternativas de movimiento y 
contacto con nuevos materiales de acuerdo a las experiencias individuales de las niñas y niños. En este sentido las estudiantes 1 y 2 señalaron:

Como he podido observar, los/as niños/as se desarrollan libremente, pero están sometidos a unos estímulos externos que la maestra les da, como hemos estudiado en clase. Deesta forma, la maestra saca de forma ordenada y a conciencia ciertos materiales que estaban guardados, algunos son demandados por los propios niños/as. Estos materiales son colocados en el aula para que puedan ser utilizados y manipulados de forma libre por los niños y niñas. (Estudiante 1)

Este método se basa en presentar un estímulo y esperar respuestas del niño/a, a las cuales se vuelven a plantear propuestas, obteniendo así nuevas respuestas con las que formulamos los objetivos alcanzados en esa sesión. Por ejemplo, cuando la primera niña que he observado estaba quieta en la cabaña de palos, la maestra ha dejado una caja con ladrillos de plástico para ver si obtenía nuevas respuestas, sin ella decirles qué hacer con ellos. (Estudiante 2)

En esa línea de atención y participación de la maestra en la sesión, las estudiantes detectaron que el profesorado estaba pendiente de promover interacciones entre el estudiantado, proponer otras actividades, y darles seguridad con su presencia, en caso de haber sido necesario. Por ejemplo, así lo resaltaron en el documento de reflexión las estudiantes 3 y 4:

La maestra en todo momento está atenta y observa las situaciones que desarrollan los niños y niñas. Construye el ambiente para que sea lo más favorable para los niños/as y reparte el material para la libre acción e interacción de estos mismos. Responde a las demandas de los niños/as. Sus sesiones no están para nada sistematizadas, cada semana es llevada a cabo de una forma distinta, pero con el mismo objetivo de desarrollar, mediante el juego, el desarrollo cognitivo, social, motor y afectivo del niño/a. (Estudiante 3)

La maestra ha introducido más cosas por la clase (aros y telas). Se ha puesto a jugar con una niña y con una tela (la niña encima de la tela y la profe la arrastraba por la clase). Al ver este comportamiento, muchos niños han imitado su acción y se han unido al juego. Cuando la maestra ha visto que los niños reaccionaban, les ha dejado solos para que experimentasen ellos solos. Es decir, ha presentado el estímulo y ha esperado la respuesta. (Estudiante 4)

\section{Respecto a la relación de los contenidos con el currículo global de la Educación Infantil}

Para el profesorado universitario que expone esta experiencia educativa universitaria, fue muy importante que la visita, observación y reflexión se convirtiese, además de en un momento interesante y novedoso, en uno como un fin educativo, a través de reflexionar sobre la relación 
doi: http://dx.doi.org/10.15359/ree.23-3.15

URL: http://www.una.ac.cr/educare

CORREO: educare@una.cr

de los contenidos teóricos y prácticos vistos en el aula universitaria, con los comportamientos, actitudes y acciones observadas en el aula de Educación Infantil. De esta manera, se ha detectado del análisis de los documentos de reflexión elaborados por el estudiantado, que las estudiantes han sido competentes para relacionar de manera global los contenidos del currículo de Educación Infantil con la práctica psicomotriz observada. En este sentido, la categoría está dividida en tres subcategorías respecto a contenidos teóricos propios de la psicomotricidad; contenidos teóricos propios de la estructura de la sesión; y finalmente, contenidos relacionados con otras áreas de conocimiento, pero vinculados con el currículo de cuerpo y movimiento. Aquí, el profesorado estableció claras relaciones entre contenidos y propuesta psicomotora cuando explicó las sesiones al alumnado, explicación previa a la observación por parte del alumnado universitario.

\section{Respecto a la relación de contenidos teóricos vinculados con la motricidad infantil}

Vaca-Escribano (2000) señaló cómo podían ser percibidas las prácticas curriculares para detectar comportamientos que, desde la visión del profesorado, contribuyen a que se presente un proceso de reflexión sobre la acción a partir de las sesiones visualizadas. Se estaría contribuyendo a unir la teoría con la práctica y la reflexión que esto implicará por parte del futuro profesorado. En este sentido, las estudiantes 2 y 8 reflexionaron de la siguiente manera.

Un ejemplo de esta fase sería cuando la maestra sacó una tela y una alumna se sentó en ella; la maestra pedía ayuda y los niños/as en vez de ayudarla a tirar de la tela, se sentaban en ella. Aquí empezó un juego que la maestra no había previsto y ella estuvo unos tres minutos compartiendo ese momento de risas y diversión con ellos/as. Otro momento de interacción directa fue cuando un niño tenía miedo de tumbar un tubo de colchoneta y la maestra le proporcionó su ayuda para que lo consiguiera. (Estudiante 2)

Por tanto, el movimiento de esta sesión es entendido, tal y como dice P. J. Arnold, como impredecible, cambiante, único, basado en la interacción, en la construcción conjunta de un significado. (Estudiante 8)

\section{La relación de conocimientos teóricos sobre la estructura de la sesión}

Además de los contenidos teóricos vinculados con escuelas de pensamiento y perspectivas curriculares que las estudiantes relacionaron con la práctica observada, se ha detectado, también, que las estudiantes relacionaron aspectos vinculados con la didáctica y, de forma más precisa, con la organización de la sesión y la forma de estructurarla; aspectos curriculares que habían sido trabajados en la puesta en práctica de sesiones de psicomotricidad en el aula universitaria. Un ejemplo de esto se puede observar en las interpretaciones de las estudiantes 7, 9 y 10. 
Por una parte, hemos visto las partes de una sesión: encuentro, construcción del aprendizaje y despedida. Y bajo mi punto de vista, la maestra de la sesión analizada, lo ha respetado notoriamente ya que la primera parte se ha llevado a cabo a través de una asamblea; la segunda parte, mediante el juego libre; y la tercera parte, mediante una relajación y la asamblea (reflexión) final. (Estudiante 7)

La primera mitad de la sesión diría que ha llevado a cabo el método centrado en el niño/a porque cada uno y una de ellos han tenido la libertad de elegir el material con el que jugar, los compañeros con los que compartirlos, los juegos desarrollados, etc. Además, he podido observar cómo los niños y las niñas iban de un lugar para otro respetando el espacio invadido por los compañeros/as y a la vez, interactuando con ellos". (Estudiante 9)

No obstante, también se trabaja el método centrado en la interacción, pues a pesar de que la motivación surge del propio niño, la maestra interviene para reconducir la sesión, dependiendo de la respuesta que ha dado el niño ante el estímulo. Por ejemplo, cuando los niños están en la casita sin hacer nada y ella introduce la construcción, o cuando ve que una niña no realiza ninguna actividad e introduce un nuevo juego con la tela. Así pues, se tiene en cuenta las características individuales de cada alumno/a, pues te centra en sus intereses personales. (Estudiante 10)

\section{Reflexiones en torno a otros bloques de contenido de la asignatura "Didáctica de la Educación Física de la Educación Infantil"}

Entre los contenidos trabajados, cabe destacar que las estudiantes han sido capaces de identificar otras temáticas trabajadas a lo largo del curso académico. Así, por ejemplo, la promoción de la igualdad de género y la forma en que se reproduce en el currículo de Educación Infantil. Lo más significativo es que, el análisis y estudios teóricos realizados en el aula, permitieron analizar la presencia de estereotipos transmitidos ya en edad tan temprana como de 3 a 6 años. Por ejemplo, las estudiantes 5 y 8 señalaron:

Una de las cosas que más me ha llamado la atención es el sexismo presente en los juegos. He notado esta situación, cuando un grupo de niñas estaba jugando con los ladrillos a un espectáculo, concretamente a ser bailarinas. Entonces, este grupo de niñas no ha permitido que un niño jugar por ser un juego de niñas. (Estudiante 5)

Esta acción nos demuestra una vez más que el sexismo se plasma en los juegos de nuestros niños. Justamente, las niñas que rechazan al niño llevaban infinitas prendas de su ropa y complementos en rosa: Calcetines, mallas, suéter, pinzas y adornos del pelo... ¿Casualidad? La escuela debe actuar contra la presión social ante estos comportamientos. (Estudiante 8) 
doi: http://dx.doi.org/10.15359/ree.23-3.15

URL: http://www.una.ac.cr/educare

CORREO: educare@una.cr

En términos generales, la detección de estas categorías, tal y como se han planteado, nos ha permitido encontrar que el estudiantado supiera relacionar la teoría aprendida con lo que estaban presenciando en los distintos centros educativos visitados. Así, pudieron armarse de argumentos para abogar por prácticas educativas no sexistas, destinadas a fomentar la igualdad de género real y efectiva. Así lo señalaron las estudiantes 1 y 5 :

También me gustaría destacar del texto de Vygotski, "El papel del juego en el desarrollo del niño", y que he podido observar, que todo juego contiene reglas de conductas. Como hemos visto en clase, para el autor, siempre que se produzca una situación imaginaria en el juego habrá reglas, pero no aquel tipo de reglas que se formulan por adelantado y que van cambiando según el desarrollo del juego, sino reglas que desprenden de la misma situación imaginaria. También respecto a la libertad en la acción del niño/a, decir que el niño/a no es libre de determinar sus propias acciones cuando juega ya que su conducta está determinada por las condiciones en las que se desarrolla la actividad: espacio, materiales, compañeros/as, maestra... (Estudiante 1)

Se interesa por el juego simbólico. El grupo de chicas no lo deja ser partícipe por afirmar que jugaban a un juego de chicas. Por reacción, otra vez a la pelota. (Estudiante 5)

\section{Respecto a la niña o niño que ha sido sujeto de la observación y la interacción de la estudiante con la maestra}

Un elemento fundamental de los procesos de observación en el aula de psicomotricidad, y reforzado como contenido en el aula universitaria, son las respuestas a los estímulos que se presentan en los momentos de encuentro, construcción del aprendizaje y despedida, esta es la autonomía en la cual las niñas y los niños participan construyendo y recreando su propio proceso de aprendizaje. En este caso, del análisis de los documentos de reflexión realizados por cada estudiante que visitó las aulas, se ha detectado que aquellas que habían sido consideradas competentes en la detección de las categorías citadas en epígrafes anteriores se habían mostrado especialmente atentas al comportamiento individual de cada niña y niño, sobre todo, en cuanto a detalles que implicaban mucha empatía, mucha percepción de elementos del área de desarrollo socioafectivo, como puedan ser el carácter extrovertido, su sensación de dependencia, sus gestos y reacciones no verbales. Así lo señalaron las estudiantes 1 y 4 :

Mi niño es muy atento y educado. Es activo y extrovertido y siempre demanda la atención del adulto para la creación de nuevas situaciones que pueda llevar a cabo. Su maestra me comenta que en el aula es siempre el que tiene la palabra en la boca y quiero hacerlo todo enseguida y el primero. En la representación se ha auto dibujado junto con sus dos perros y papeles alrededor. Comenta que lo de los papeles le ha encantado. (Estudiante 1) 
En general, a lo largo de la sesión, la interactuación de Aarón con los materiales se ha centrado en doso tresy se ha repetido a lo largo del tiempo. Hajugado con los bloques, la pelota saltarina y los papeles; $y$ al final del todo, con una tela naranja. Sin embargo, no ha hecho caso al resto del material. En cuanto a las personas, la mayoría del tiempo buscaba la complicidad de la tutora. En algún momento puntual, ha interactuado con Ma José, y en contadas ocasiones ha jugado con algún niño o niña. (Estudiante 4)

En esta experiencia, el profesorado del centro escolar estuvo muy implicado y departió gustosamente con todo el estudiantado, expresando no solo sus opiniones sobre la propia intervención psicomotora, sino acerca de otras alternativas estudiadas durante su formación inicial. Además, comentaron las ayudas recibidas para adecuar el aula de psicomotricidad, tanto por parte del centro, como del alumnado de educación primaria, que lo asumía como una responsabilidad que posibilitaba un trato más cercano con el profesorado. Así lo resaltaron, por ejemplo, las estudiantes 4 y 6 .

El año pasado, cuando llegó al colegio, el aula de psicomotricidad era un almacén, lleno de todos aquellos objetos que nadie sabía dónde colocar. Ella lo arregló y lo ha convertido en un aula de psicomotricidad, adecuando el espacio a sus necesidades. Cuando aceptó encargarse de las sesiones de psicomotricidad del ciclo de infantil, puso dos condiciones que fueron aceptadas por el centro. La primera de ellas fue poder comprar algo de material; y la segunda, que las educadoras de infantil estuvieran con ella durante las sesiones. (Estudiante 4)

Antes de que entrasen los alumnos/as en el aula, la maestra nos comentó cual era la estructura de su clase. Primero desarrollaban juego libre, más tarde utilizaban los rincones y finalmente pasa a lo cognitivo, preguntando a los niños/as que representasen lo que habían hecho en la sesión de psicomotricidad. (Estudiante 6)

En este marco, coincidimos con Álvarez y Hevia (2013), en el sentido de que el nuevo modelo de formación del profesorado exige que los entes involucrados en los procesos de enseñanza-aprendizaje, en todos los niveles y sectores, se conozcan mejor y colaboren para favorecer una educación superior de máximo nivel.

\section{Respecto a las limitaciones detectadas y posibles explicaciones en la estructura y desarrollo de la sesión}

Las consideraciones del estudiantado al respecto resultaron interesantes, porque se les estaba mostrando la necesidad del alumnado de comunicar qué había hecho, qué había sentido durante la sesión de psicomotricidad. En otras palabras, fueron capaces de detectar 
doi: http://dx.doi.org/10.15359/ree.23-3.15

URL: http://www.una.ac.cr/educare

CORREO: educare@una.cr

oportunidades de retroalimentación, así como limitaciones en esas oportunidades, mediante distintas posibilidades de expresión, demostrado de gran utilidad para empezar a comprender y comprenderse. Así lo señaló la estudiante 2.

Sin embargo, el momento de despedida de la sesión no hasido el esperado, ya que simplemente han dicho lo que más les ha gustado y en clase hemos dado que debemos hacerles ser conscientes a los niños y las niñas de sus acciones a lo largo de la sesión, y que enseñen al resto de compañeros algún momento clave que haya sido significativo para ellos. Esto les ayuda no solo a darse cuenta de qué han hecho en ese tiempo y ese espacio, sino a cómo lo han llevado a cabo. Este fallo pudo haberse dado por la falta de tiempo o por otros condicionantes ya que la maestra nos dijo que muchas veces realizaban un dibujo y lo explicaban al resto de la clase. (Estudiante 2)

Por su parte, la estudiante 3 señaló el choque entre el concepto teórico aprendido en clase y la realidad educativa del centro visitado. Esto les permitió comprender cómo es necesario ajustar lo aprendido a lo necesario para el estudiantado, para sus necesidades educativas. Debían aprender a discernir si lo aprendido podía aplicarse para mejorar la realidad de la práctica educativa analizada en ese centro.

También, como hemos visto, al terminar la actividad, no se marchan directamente a casa, sino que se sientan en un círculo y se comienza a recordar aquello que se ha hecho. La profesora nos explicó que algunas veces hacen un dibujo para plasmar aquello que han realizado durante la sesión (pero esta vez no daba tiempo). En su lugar, de uno en uno, decían qué era lo que más les había gustado. Sin embargo, en clase hemos visto que para que asimilen mejor lo conceptos no vale con decirlo verbalmente, sino que es recomendable decir que lo repitan en ese momento para que todos lo vean y lo asimilen mejor. Pero, como he dicho, en muchas ocasiones, el tiempo juega una mala pasada y no te permite hacer todo lo que tienes planeado (así pues, se puede observar la capacidad de la maestra para reconducir la clase). (Estudiante 3)

Sin embargo, no todas las sesiones analizadas mostraron ese último momento de reflexión y, en este caso, lo importante fue cómo el alumnado se mostró consciente de ello y de lo importante que le parecía la inclusión de dicho momento para garantizar el aprendizaje de lo vivenciado en la sesión de psicomotricidad.

No se llegan a apreciar las 3 partes de la sesión. El momento de recibimiento es escaso. Y después la parte de la actividad dura toda la sesión, sin dejar tiempo a la parte de despedida. Tal ycomo hemos visto, ese momento asambleario es esencial para completar la adquisición de conocimientos y no se ha dado. Hubiera estado interesante elaborar una sesión para que nos contaran a qué han jugado, cómo, cómo se han movido, cómo se han sentido etc. (Estudiante 5) 
El establecer oportunidades de juego y movimiento, siguiendo tiempos y orden, o bien el presentar pequeños rincones de juego e interacción, son cuestiones que fueron especialmente valoradas, tanto en positivo como en negativo por parte del estudiantado. Consideraron que la experimentación libre les parecía más oportuna y adecuada para vivenciar la experiencia motriz; en este sentido, la teoría estudiada comentaba diferentes opciones, igualmente validadas y exploradas. Estamos de acuerdo con las conclusiones a las que llegaron Mérida et al. (2011) en el sentido de que las oportunidades de vincular el trabajo de las escuelas con la formación del profesorado y, en especial, de maestras y maestros en Educación Infantil, son elementos fundamentales y necesarios que justifican la continuación de trabajo conjunto para que el futuro profesorado en ejercicio desarrolle un conocimiento experiencial que le permita comprender el conocimiento de la asignatura; pero, sobre todo, comprender la forma de ejercer dicho conocimiento en sus prácticas curriculares de manera positiva. Así también lo señala la estudiante 5:

Otro aspecto a comentar, es que no se plantean propuestas de juegos a los niños. Tal y como hemos visto, esta interacción es importante para crear significado del movimiento y para introducir mejoras a nivel motriz. En el niño observado, esto se ha observado muy bien, puesto que, al no haber interacción, el niño se ha dedicado la gran parte de la sesión a jugar con la pelota. Tal vez, con interacción le hubiéramos podido plantear alternativas con la pelota o incluso alternativas de material. (Estudiante 5)

\section{Respecto a la utilidad y el agrado de haber participado en la sesión de observación}

Un aspecto importante que se ha detectado en esta experiencia educativa es que, a diferencia de lo que se cree, el periodo de prácticas en la formación del profesorado no es el espacio en el cual las futuras maestras y maestros tienen contacto con la realidad escolar. Algunas veces, como se verá a continuación, puede ser que en los periodos de prácticas ciertas temáticas y procesos tan importantes para la formación del profesorado hayan pasado desapercibidos y que, por tanto, sea en experiencias de este tipo en donde el estudiantado puede tener un primer contacto con estas prácticas curriculares. En este marco, Mérida et al. (2011) señalaron que la experiencia de innovación respecto a la percepción de la implementación de un programa sobre metodología por proyectos de trabajo, en el marco de colaboración entre la universidad y las aulas escolares, genera, en el estudiantado y el profesorado, altos niveles de aprendizajes relacionados con las competencias docentes en la educación infantil. En este sentido, esta experiencia también consiguió resultados similares y, sobre todo, mostró a las futuras docentes la clara relación entre la teoría del aula y la práctica diaria, como lo señala la estudiante 2. 
doi: http://dx.doi.org/10.15359/ree.23-3.15

URL: http://www.una.ac.cr/educare

CORREO: educare@una.cr

Y para acabar, me gustaría decir que me ha encantado esta experiencia nueva de analizar detalladamente una sesión de psicomotricidad porque me ha hecho ver de una manera distinta, pero más clara, los conocimientos vistos en clase. Además, me ha acercado a esa perspectiva práctica que tan extraña veíamos al principio, la cual me ha enseñado que cuando la sesión termina con una distribución de los materiales totalmente distinta a la del inicio y que cuando ninguna sesión es igual a la anterior, significa que se ha entendido y desarrollado la verdadera esencia de la educación física. (Estudiante 2)

De esta forma, se observa cómo la verdadera esencia de las sesiones de psicomotricidad, en algunos de los centros visitados, es una de las ideas importantes con las que el estudiantado debía quedarse. Cuando educamos a través del cuerpo, pero sobre todo, en la vivencia de las propias posibilidades motrices que se construyen en interacción con el entorno (materiales, niñas niños y profesorado) se realiza con base en unos principios y objetivos que, en el caso de la Educación Infantil, van encaminados al control de su cuerpo, a una consciencia corporal que viene facilitada por el dominio de sus destrezas motrices básicas y por el conocimiento de sus propias posibilidades motrices y de los otros seres en un ámbito de respeto a la diversidad. Entre esos objetivos y principios está la consecución de un autoconcepto positivo respecto a las propias posibilidades motrices (Conselleria de Educación, 2008), justo lo que observaron que podría conseguirse gracias a la actitud de la docente observada. Así lo reflejó la estudiante 5:

Una actuación positiva por parte de la maestra es la constante retroalimentación. Considero que este hecho ayuda a que los niños no se cohíban a la hora de realizar determinados movimientos y los invita a continuar con la actividad. (Estudiante 5)

Por otra parte, tal como sostuvo Vaca-Escribano (2000), el registro sistemático de lo que ocurre en las sesiones de motricidad y, especialmente durante el momento de actividad motriz, permite a quienes participan en el proceso de observación de las sesiones "retomar las tareas comunes, derivadas o propuestas, desarrolladas a lo largo del curso escolar... para hacer un análisis retrospectivo sobre su oportunidad pedagógica y planificar el curso siguiente con mayor fundamento" (Vaca-Escribano, 2000, p. 121). Una reciente investigación señaló que el estudiantado del Grado de Magisterio en Educación Infantil demanda un mayor componente de práctica en la carrera (Gil-Madrona et al., 2016). Tal como apuntan Gil-Madrona et al. (2016), una buena relación entre los contenidos teóricos de la instrucción y su conexión con la puesta en práctica de dichos contenidos son claves acerca de la forma a través de la cual se imparten los contenidos. Así se observó en los comentarios de las estudiantes, por ejemplo, la estudiante 3:

En conclusión, creo que esta sesión de motricidad ha resultado muy interesante e instructiva, pues al no haberla visto nunca, no tenía ninguna referencia. Además, pienso que el método que utiliza (centrado en el niño y en la interacción) es idóneo para que los niños/as se desarrollen por completo según sus intereses y aptitudes. (Estudiante 3) 
De esta manera, estamos de acuerdo con el planteamiento de Álvarez y Hevia (2013), en el sentido de que el acercamiento a la realidad de las escuelas es fundamental en la formación del profesorado. En esta experiencia, gracias a los documentos de reflexión, se ha detectada que las estudiantes no solamente tienen un contacto verbal con el profesorado de los centros, sino que conocen como se construyen las prácticas curriculares, y que, en ese proceso, existen oportunidades y, en algunos casos, barreras que hacen parte de la realidad escolar.

Finalmente, se quiso identificar cuál había sido la percepción de las dos maestras que participaron en la experiencia. Se obtuvieron datos interesantes de las entrevistas realizadas a las dos maestras que facilitaron el acceso a sus aulas. De igual forma, los datos obtenidos en estas entrevistas fueron especialmente relevantes para concretar y mejorar futuros proyectos de innovación. Por ejemplo, una maestra señaló:

Por la relación que estoy teniendo con vosotros, y ya tan sólo ver el interés que tenéis por cambiar el modelo este de la maestra de Infantil que no haga psicomotricidad, creo que es un bien que se van a beneficiar los colegios y eh h yo me imagino que estáis valorando sobre todos los profesores de Educación Física, que si no se empieza desde la base ehh Es muy importante, ¿no? Entonces eso también es importante, el aprendizaje que yo he hecho, porque si no empiezas por infantil, luego las dificultades que tienen en el aprendizaje de todo ehh ahh son muy grandes y, por eso, a mí me apasiona tanto la psicomotricidad, porque dices a través de la psicomotricidad se pueden trabajar tantas cosas que cuando llegan a primaria todos son beneficios. (Maestra 1 del centro escolar)

Tal como se observa, la maestra remarca la importancia de trabajar desde esas directrices en Educación Infantil para que, en la etapa siguiente de Educación Primaria, todo fuesen ventajas para el alumnado. Pero, además, se resalta de la entrevista que la maestra percibe, igual que el profesorado universitario que esto escribe, que no existe una verdad absoluta en el tratamiento de la psicomotricidad en Educación Infantil, sino que esta se construye de la interacción entre quienes participan del proceso educativo.

Por otra parte, la maestra 2 señaló:

Pues materiales que he ido encontrando, igual que la música, tampoco siento que aprendiera mucho en Magisterio, y un poco me busqué la vida buscando material, pero es que se lo pides a cualquier maestra a casi cualquier maestra y da respeto ehh, fíjate que deberías estar, sentirnos preparadas para hacer psicomotricidad, música y son áreas que por lo menos antes no no se daba la importancia y no estamos tan preparadas, de hecho la gente se acomoda que alguien le imparta esas áreas... (Maestra 2 del Centro Escolar) 
doi: http://dx.doi.org/10.15359/ree.23-3.15

URL: http://www.una.ac.cr/educare

CORREO: educare@una.cr

De la misma forma, del análisis de esta entrevista se desprende que la formación universitaria respecto a la psicomotricidad es clave en la seguridad que cada maestra tiene frente a sus prácticas curriculares. $Y$ es en este contexto en donde la presente experiencia innovadora quiere dirigirse: trabajar conjuntamente con las escuelas para que las maestras en formación tengan la oportunidad de ver aulas reales en las que se trabajan las posibilidades de movimiento y de autonomía personal.

En definitiva, las verdades diversas de la forma en que se construye el cuerpo infantil y sus posibilidades de movimiento trabajan constantemente en pie de igualdad, y se considera que esta experiencia innovadora abre la puerta para seguir explorando, conjuntamente con el profesorado de los centros escolares, el estudiantado y las facultades de formación del profesorado, en la construcción de las prácticas curriculares que fomenten la formación integral de las niñas y niños entre los 3 y los 6 años.

\section{Conclusiones}

El objetivo del presente estudio consistió en analizar y evaluar la puesta en práctica de una experiencia centrada en la didáctica de la motricidad infantil, mediante la participación de un grupo de maestras en ejercicio, de estudiantes y de profesorado universitario dentro de la asignatura de "Didáctica de la Educación Física de la Educación Infantil" del plan de estudios del Grado de Maestra-o en Educación Infantil. De los resultados obtenidos, se pudieron extraer distintas categorías que permitieron cualificar las experiencias, sus elementos más habituales y característicos, las relaciones entre docente-alumnado, las propuestas que podían funcionar con unos u otros perfiles.

En particular, del análisis de los relatos se extrajeron los siguientes resultados. Se reagrupó la información en cinco categorías, a saber: respecto a la forma en que la maestra facilita la interacción a través del uso de materiales; respecto a la relación de contenidos vistos en la universidad con la práctica real del aula; respecto a la niña o niño que ha sido sujeto de la observación y la interacción de la estudiante con la maestra; respecto a la detección de limitaciones de la sesión y posibles explicaciones y, finalmente, respecto a la utilidad y el agrado de haber participado en la sesión de observación. Posteriormente, utilizando esas cinco categorías profundizamos el análisis de las categorías con mayor presencia de alusiones a los bloques de contenidos de la asignatura vinculadas con la creación, ejecución y evaluación de las sesiones de psicomotricidad, por una parte, y, por otra, respecto a la detección de fortalezas y debilidades que, fruto de la reflexión, pueden ser mejoradas o reforzadas.

En general, el futuro profesorado pudo presenciar in situ las diferentes concepciones de la psicomotricidad revisadas teóricamente en el aula, principal aporte del citado proyecto que se presenta en este documento. De modo que, con la puesta en práctica del proyecto de innovación 
realizado, se ha querido fortalecer este propósito a través de una mirada en la cual no es el sistema escolar quien se acerca a las aulas universitarias, sino son las aulas universitarias y, concretamente, el estudiantado y el profesorado quienes se acercan a las aulas del sistema escolar.

Esta experiencia fue supremamente positiva y dio la oportunidad de reflexionar sobre mejores prácticas docentes para el futuro. Incluso, como fruto de la misma, en el mes de julio de 2017, se organizó una Jornada para socializar resultados provisionales del proyecto, que fue en total éxito de participación: asistieron las maestras que facilitaron el acceso a los Centros Escolares, alumnado implicado y profesorado en activo interesado. Se contó con la ayuda del Servicio de Formación del Profesorado de la Universidad de Valencia, tanto del área de formación como de innovación. En los últimos años, no se había realizado en la Universidad de Valencia y en la Facultad de Magisterio, una jornada centrada en la temática de la psicomotricidad, particularmente, para el profesorado de Educación Infantil. No obstante los elementos positivos, también hemos detectado situaciones a mejorar. Entre ellas se destaca la necesidad de ampliación a nuevos contactos con otros centros escolares, una mayor motivación a otras estudiantes para la participación en la experiencia innovadora, y una evaluación de la necesidad de trabajar conjuntamente con otras asignaturas del mismo curso académico, que permitan al estudiantado relacionar los contenidos y procesos vinculados con la formación integral de la niña y el niño entre los 0 y 6 años. De los resultados del presente proyecto de innovación y en particular, del análisis cualitativo de la experiencia vivida por el estudiantado en formación, señalamos que hay que continuar con el trabajo de unir dos mundos, el universitario y el escolar, en aras de fomentar una formación de calidad.

En definitiva, con los resultados del análisis de esta experiencia innovadora, se estaría en capacidad de señalar que las visitas a los centros escolares para la observación y reflexión de sesiones de psicomotricidad infantil pueden favorecer la formación del futuro docente de Educación Infantil. Se ha considerado que el estudiantado universitario podrá tener un contacto más cercano con la forma en que se trabaja la psicomotricidad en las aulas de Educación Infantil para desarrollar una actitud crítica hacia el papel de la educación motriz en el currículo de dicha etapa educativa, lo que está claramente relacionado con las diferentes competencias de la asignatura (Universitat de València, 2017a). En este trabajo se ha presentado una metodología docente universitaria que intenta cumplir con los grandes retos del nuevo papel que cumple la formación universitaria, así como la necesidad de implementar momentos para la interacción que favorezcan la comunicación fluida entre la escuela y la universidad, y promuevan el trabajo continuo de competencias transversales y específicas del estudiantado universitario en el nuevo EEES (Valero, Aramburu, Baños, Sentí y Pérez, 2007).

Esta experiencia pretende, por tanto, ser un nuevo aliciente en la búsqueda de alternativas para llevar a cabo procesos de acompañamiento con el estudiantado, con el propósito de acercarle a la realidad de las aulas escolares de educación infantil. 
doi: http://dx.doi.org/10.15359/ree.23-3.15

URL: http://www.una.ac.cr/educare

CORREO: educare@una.cr

\section{Agradecimientos}

Este proyecto ha sido realizado gracias a la ayuda recibida por el Vicerectorat de Polítiques de Formació i Qualitat Educativa de la Universitat de València en el marco de la convocatoria de Projectes Innovació Educativa 2016/2017 (Referencia UV-SFPIE_RMD16-418238). Agradecemos a las maestras de los centros escolares por compartir sus prácticas curriculares en el marco de esta experiencia innovadora.

\section{Referencias}

Álvarez, C. y Hevia, I. (2013). Posibilidades y límites de la relación teoría-práctica en la formación inicial del profesorado. Cultura y Educación, 25(3), 337-346. doi: https://doi. org/10.1174/113564013807749759

Blanco, N., Molina, M. D. y López, A. (2015). Aprender de la escuela para dar vida a la universidad. Revista Interuniversitaria de Formación del Profesorado, 82(29.1), 61-76. Recuperado de https://www.redalyc.org/html/274/27439665005/

Bueno-Moral, M. L., Manchón-Ruíz, J. I. y Moral-García, P. (1990). Educación infantil por el movimiento corporal: $2^{\circ}$ ciclo, 3 a 6 años:lidentidad y autonomía personal. Libro de fichas. Madrid: Gymnos.

Conselleria de Educación (2008). Decreto 38/2008, de 28 de marzo, del Consell, por el que se establece el currículo del segundo ciclo de la Educación Infantil en la Comunitat Valenciana. DOGV (03/04/2007), núm. 5734, referencia 2008/3838, pp. 55018-55048.

Contell-Lahuerta, S., Molina-García, J. y Martínez-Bello, V. E. (2017). Niveles y patrones de actividad física en sesiones de motricidad infantil basadas en el juego libre. Sportis. Scientific Journal of School Sport, Physical Education and Psychomotricity, 3(2), 303-322. doi: https://doi.org/10.17979/sportis.2017.3.2.1891

Gil-Madrona, P. (2003). La educación física en el acto didáctico de la educación infantil desde la perspectiva de la universidad, los jardines de infancia y los colegios de infantil y primaria. Revista de la Facultad de Educación de Albacete, 18, 93-131. Recuperado de https://previa. uclm.es/ab/educacion/ensayos/pdf/revista18/18 6.pdf

Gil-Madrona, P., García-Gómez-Heras, S., Hernández-Barrera, V., López-de-Andrés, A., LópezGómez, L., Fernández-García, H., Díaz, A. y Carrasco-Garrido, P. (2016). Comportamientos del profesor universitario y éxito académico de los estudiantes de los grados de maestro de infantil y primaria. Anales de psicología, 32(3), 847-854. doi: http://dx.doi.org/10.6018/ analesps.32.3.221061 
González, J. y Wagenaar, R. (Eds.). (2003). Tuning educational structures in Europe. Informe final. Proyecto piloto - Fase uno. Bilbao: Universidad de Deusto. Recuperado de http:// tuningacademy.org/wp-content/uploads/2014/02/TuningEUI Final-Report SP.pdf

Howie, E. K., Brewer, A. E., Dowda, M., Mclver, K. L., Saunders, R. P. y Pate, R. R. (2016). A tale of 2 teachers: A preschool physical activity intervention case study. Journal of School Health, 86(1), 23-30. doi: https://doi.org/10.1111/josh.12352

Ibarra, M. S. y Rodríguez, G. (2011). Aprendizaje autónomo y trabajo en equipo: Reflexiones desde la competencia percibida por los estudiantes universitarios. Revista Electrónica Interuniversitaria de Formación del Profesorado, 14(4), 73-85. Recuperado de https://www. redalyc.org/articulo.oa?id=217022117006

Martínez-Bello, V., Bernabé, M., Molina, J. y Estevan, I. (2017). Psicomotricidad infantil: De las aulas universitarias a las aulas de educación infantil (UV-SFPIE_RMD16-418238). Proyecto de Innovación de la Universidad de Valencia, Vicerrectorado de Políticas de Formación y Calidad Educativa.

Mauri, T., Onrubia, J., Colomina, R. M. y Clarà, M. (2016). Espacios de formación compartida escuela-universidad en el prácticum. Aula de innovación educativa, 256, 12-16.

Mérida, R., González, E. y Olivares García, M. (2011). Adquisición de competencias profesionales del alumnado de Magisterio de Educación Infantil a través de una red de colaboración escuela-universidad. Revista de Investigación en Educación, 9(2), 184-199. Recuperado de https://dialnet.unirioja.es/servlet/articulo?codigo $=4731554$

Ministerio de Educación y Ciencia. (2006). Propuestas para la renovación de las metodologías educativas en la Universidad. Madrid: Secretaría General Técnica. Recuperado de http:// www.etsii.upct.es/pdfs/propuesta renovacion crue.pdf

Pérez, M. J. (2011). La innovación educativa. Pedagogía Magna, 10, 93-97. Recuperado de https:// dialnet.unirioja.es/ejemplar/277801

Rosales, C. (2013). Competencias específicas curriculares que ha de adquirir el estudiante del título de grado de maestro. Profesorado. Revista de Currículum y Formación de Profesorado, 17(3), 73-90. Recuperado de https://www.redalyc.org/pdf/567/56729527005.pdf

Silva-Moreira, M., Neves de Almeida, G. y Moreira-Marinho, S. (2016). Efectos de un programa de psicomotricidad educativa en niños en edad preescolar. Sportis: Revista Técnico-Científica del Deporte Escolar, Educación Física y Psicomotricidad, 2(3), 326-342. doi: https://doi. org/10.17979/sportis.2016.2.3.1563 
doi: http://dx.doi.org/10.15359/ree.23-3.15

URL: http://www.una.ac.cr/educare

CORREO: educare@una.cr

Taylor, S. y Bogdan, R. (1996). Introducción a los métodos cualitativos de investigación. La búsqueda de significados. Barcelona, España: Paidós Básica.

Teixeira-Costa, H. J., Abelairas-Gomez, C., Arufe-Giráldez, V., Pazos-Couto, J. M. y Barcala-Furelos, R. (2015). Influence of a physical education plan on psychomotor development profiles of preschool children. Journal of Human Sport and Exercise, 10(1), 126-140. Ddi: https://doi. org/10.14198/jhse.2015.101.11

Universitat de València (2017a). Guía docente asignatura Didáctica de la Educación Física de la Educación Infantil. Valencia: Universitat de València.

Universitat de València (2017b). Competencias específicas del título de Maestra-o en Educación Infantil. Valencia: Universitat de València.

Vaca-Escribano, M. (2000). Reflexiones en torno a las posibilidades educativas del tratamiento pedagógicodelocorporalenel segundociclo deeducación infantil.Revista Interuniversitaria de Formación del Profesorado, 37, 103-120. Recuperado de https://dialnet.unirioja.es/ servlet/articulo?codigo $=118061$

Vaca-Escribano, M. y Medina-Vaquero, M. (1997). Tratamiento pedagógico de lo corporal en educación infantil: Construir el significado educativo de una práctica desde la interpretación y explicación compartida de lo ocurrido. Revista Interuniversitaria de Formación del Profesorado, 1, 1-6. Recuperado de https://dialnet.unirioja.es/servlet/ articulo?codigo $=2783863$

Valero, M., Aramburu, J., Baños, J.-E., Sentí, M. y Pérez, J. (2007). Introducción de un portafolio para fomentar las competencias transversales de los estudiantes universitarios. Educación Médica, 10(4), 50-57. doi: https://doi.org/10.33588/fem.104.8

Vázquez, G. (2016). El mito de la innovación tecnológica: ¿Se reduce toda innovación educativa a la innovación tecnológica? En Grupo SI(e)TE. Educación (aut.), Repensar las ideas dominantes en educación (pp. 163-174). Santiago de Compostela: Tórculo Edicions.

Zabalza, M. A. (2008). El trabajo por competencias en la enseñanza universitaria. En I. Rodríguez (Ed.), El nuevo perfil del profesor universitario en el EEES: Claves para la renovación metodológica (pp. 79-113). Madrid: Universidad Europea. 